
$\angle$ Research Square
Preprints are preliminary reports that have not undergone peer review. They should not be considered conclusive, used to inform clinical practice, or referenced by the media as validated information.

\title{
Efficacy of an 8-week Bedside Ultrasound Training Program for Surgical Residents in the Intensive Care Unit
}

\section{Kyoung Moo Im}

Department of Surgery, Seoul St. Mary's Hospital, College of Medicine, The Catholic University of Korea

Eun Young Kim ( $\square$ freesshs@naver.com)

Division of Trauma and Surgical Critical Care, Department of Surgery, Seoul St. Mary's Hospital, College of Medicine, The Catholic University of Korea

\section{Research Article}

Keywords: Surgical resident, Ultrasound, Clinical competence, Education, intensive care unit

Posted Date: March 11th, 2021

DOI: https://doi.org/10.21203/rs.3.rs-265514/v1

License: @ (i) This work is licensed under a Creative Commons Attribution 4.0 International License. Read Full License 


\section{Abstract}

\section{Background}

Bedside ultrasound has become one of the most important non-invasive and readily available diagnostic tools especially for critically ill patients. However, the current ultrasound training program is not standardized and is mostly unavailable to all surgical residents equally. The aim of this study was to assess and evaluate the effectiveness of our new training program in bedside ultrasound for surgical residents.

\section{Methods}

Postgraduate residents (years 1 to 4) from the department of general surgery in a tertiary hospital attended the newly designed, 8-week training course in bedside ultrasound for critically ill patients at the surgical intensive care unit. Didactic and experimental lectures in basic ultrasound physics and machine usage were delivered, followed by daily hands-on ultrasound training to patients. Each participant prospectively documented their ultrasound findings and completed self-assessment of ultrasound skills using a Likert scale.

\section{Results}

44 residents were enrolled in the current study and only $36.4 \%$ of them were previously exposed to bedside ultrasound experience. Following the course completion, the proficiency levels and the objective structured assessment of ultrasound skill (OSAUS) scores showed significant improvement in all elements $(P<0.001)$. The mean differences in pre- and post-course score between post-graduate years were recorded, except for proficiency in peritoneal cavity $(P=$ 0.163). Post-hoc analysis revealed that post-graduate year 2 residents showed a higher improvement in most elements. The training program showed improvement in post-course scores, whether or not residents had previous experience.

\section{Conclusions}

Our results revealed that the knowledge and confidence of surgical residents in bedside ultrasound could be improved via short-term and impact training curriculum. The authors believe that such education should be encouraged for all surgical residents to enhance competency in performing bedside ultrasound evaluation of critically ill patients.

\section{Background}

To general surgeons, the use of ultrasound has proven to be valuable in enhancing the level of care provided to patients in various settings such as emergency department, operating room, or intensive care unit (ICU) [1, 2]. It is an effective diagnostic tool for detecting free fluid in trauma patient, to facilitate rapid diagnosis of shock etiology, or determine fluid status in critical ill patients [1-3]. It can also be used for surgical interventions such as central venous catheter placement, drain insertion, aspiration of fluid collection or a wide range of other procedural guidance [4,5]. Especially in the ICU, where most of the patients are immobilized and hemodynamically unstable, bedside ultrasonography is attractive because it is noninvasive and portable, and facilitates rapid assessment of critically ill patients [6-8]. Moreover, as the proportion of the elderly patients increases, the risk of postoperative complications increases. Further, more patients are undergoing highly complex surgeries due to advances in operative technique and surgical instrumentation, which can increase the risk of complication [9]. Therefore, tools that can facilitate early identification and acquisition of real-time images bedside are the need of the hour. The American College of Surgeons (ACS) has been providing ultrasound courses in the United States, since 1996 [4]. In South Korea, the Korean Surgical Society (KSS) mandates that all residents in general surgery attain competency in the use of ultrasound for various surgeries. However, despite the obvious advantages and utility of bedside sonography, many surgical residencies still lack practical opportunities or adequate ultrasound training curriculum, possibly due to curricular time constraints, lack of equipment, and limited availability of skilled faculty [10]. It has led to minimal ultrasound training or experience in most of the surgical residencies during their training period. Therefore, the implementation of a formal and well-established training program in bedside ultrasonography is important for surgical residents in charge of patient care.

Herein, we introduced our 8-week standardized bedside sonography training program that include clinical application of skills to the actual ICU patients. Additionally, the changes in confidence of residents before and after the training and the effectiveness of the training curriculum were assessed.

\section{Methods}

\section{Participants and methods}

From March 2019 to February 2021, the residents of department of general surgery in our institution from post-graduate year-1(PGY-1) to four (PGY-4) enrolled in the study. The study involving the 8-week program in bedside ultrasound training at the surgical ICU was approved and carefully monitored by the Institutional Review Board of the Ethics Committee of our institution (KC20QISI0723). The written informed consent from the participants were obtained, and the procedures were in compliance with Helsinki Declaration. Each resident's training program consisted of a didactic presentation and practical session, involving extended-Focused Assessment with Sonography for Trauma (e-FAST) examination and a volume assessment of the patients admitted to the surgical ICU. The course was followed by a post-course survey. Each of the residents evaluated their own competency in ultrasound examination and documented the ultrasound findings of each patient. None of the data collected were linked to individual participants, so the results of assessment did not have an impact on assessing the individual participants' abilities. The ultrasound machine used for program was GE Healthcare LOGIQ P9 (Boston, MA). A convex transducer (C1-5, low frequency, 2-5 MHZ) and a linear transducer (3SC, high frequency, 1.7-4MHZ) were used for training and assessment. 
The didactic presentation was developed by the surgeon specialized in trauma and surgical critical care and the resident received about one-hour didactic lecture once a week. The lecture included basic physics of ultrasound, knobology, artifacts, e-FAST technique and a technique for assessing IVC diameter. The surgeon specializing in trauma and surgical critical care also conducted the practical sessions. The residents performed a supervised e-FAST examination and assessment of inferior vena cava (IVC) volume of patients admitted to the surgical ICU. Each resident focused on visualization of each organ (lung, liver, spleen, bladder, kidney and heart), and also identified any abnormal findings during e-FAST. During the examination, the patient was positioned in a supine to $30^{\circ}$ upright angle. The exam combined focused evaluation of five areas: chest, right upper quadrant, left upper quadrant, suprapubic and sub-xiphoid according to the standard method of e-FAST shown in Figure $1[2,6-8]$.

To assess the volume of IVC (Figure 2), either curvilinear or phased array probe were used on the subcostal window and scanned transverse image of IVC and right atrium. A probe was rotated $90^{\circ}$ to obtain the long axis of IVC. The longest and the shortest diameters of IVC were measured at 1 to $2 \mathrm{~cm}$ away from the right atrium. The M-mode scan was used to capture both the longest and the shortest diameters of IVC on a single image. IVC collapsibility index was also calculated using the following formula.

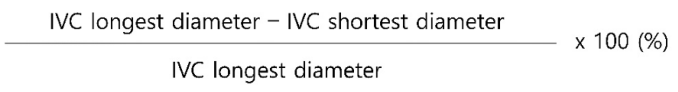

\section{Study endpoint and outcome measurement}

The primary outcomes included assessment of basic knowledge and ultrasound competency of each resident as well as the efficacy of an ultrasound training program based on the comparative evaluation score of the perceived self-confidence levels before and after the training measured on a 5-point Likert scale. The secondary outcome measures were to assess differences in the program efficacy by post-graduate year or previous experience in bedside ultrasonography.

\section{Participants' assessment}

Participating residents completed the survey to assess their own comprehension and confidence (Appendix 1). The survey was used to evaluate the objective structured assessment of ultrasound skill (OSAUS) (Appendix 2). All questionnaires were estimated using a 5-point Likert scale, and the score ranged from 1 to 5 ( 1 = not confident at all, and 5 = very confident). The Likert scale is an orderly scale and a form of closed question that is most widely used in the analysis of opinions or educational training. Its advantage relates to the absence of forced expression to elicit participants' opinions [5, 11-16]. We investigated how the residents perceived their confidence and proficiency during overall examination and queried in detail according to seven different areas of the patient's body (lung, pleural effusion, bowel, peritoneal cavity, liver, gallbladder, spleen, jugular vein, inferior vena cava). Furthermore, the resident's competency was also assessed using a Delphi's Objective Structured Assessment of Ultrasound Skills (OSAUS), which is a generic ultrasound rating scale based on international multispecialty consensus [17]. We modified the original form of OSAUS by including queries based on five elements: applied knowledge of ultrasound equipment, image optimization, systematic examination, image interpretation and documentation of examination.

\section{Statistical analysis}

All statistical analyses were conducted using SPSS statistical package software (version 21.0 for Windows; SPSS, Inc., Chicago, IL). Survey responses to questions regarding confidence ranged from 1 (not confident at all) to 5 (very confident). For the purpose of statistical analysis, responses were divided into "not confident (not confident, minimally confident, and neutral, based on scores ranging from 1 to 3 ) and "confident" (confident and very confident, based on scores from 4 to 5). To assess the differences in confidence levels we compared the demographics, previous training history, as well as other variables of residents who reported confidence with training compared with those who did not. Continuous data are presented as the mean \pm standard deviation. For continuous data, overall differences were tested by Student's t-test or ANOVA. The categorical variables were calculated using the Fisher's exact test or chisquare test. The descriptive statistics are described as means \pm standard deviation and differences were regarded as statistically significant when $P<0.05$. Multivariable logistic regression analysis was then performed to identify independent predictive factors.

\section{Result}

During the study period, a total of 44 residents from PGY1 to PGY4 completed our eight weeks of bedside ultrasound training program at the surgical ICU. Among them, only sixteen participants were experienced in bedside ultrasound before the training as shown in Fig. 3 .

The definition of an experience group is as the participants who had performed bedside ultrasound on at least five times in clinical setting prior to study enrollment. The average number of experiences of the experimental group before the study enrollment was 5.8 , whereas the average number of nonexperienced group was 0.9 . Finally, total of 4,872 ultrasound examinations with 818 patients were completed and analyzed. The mean age of patients was 67.2 years and the majority of them was from the departments of lower gastrointestinal surgery $(n=221,27 \%)$ followed by hepatobiliary-pancreas surgery ( $n$ $=180,22 \%)$. The patient demographics and sonographic findings are summarized in Table 1. 


\section{Trainee $(\mathrm{N}=\mathbf{4 4})$}

Postgraduate Year 1

2 11

3

9

3

9

4

15

Previous experience of bedside sonogram*

Experienced/Inexperienced

Number of experiences of bedside sonogram before the study enrollment

\begin{tabular}{ll} 
Experienced group & $5.8 \pm 4.3$ \\
\hline Inexperienced group & $0.9 \pm 4.3$
\end{tabular}

\section{Patient demographics $(\mathrm{N}=\mathbf{8 1 8})$}

Age

$67.2 \pm 16.3$

Sex

Male/Female

$530 / 288$

ICU HD at the time of sonogram

$6.8 \pm 7.8$

POD at the time of sonogram

Clinical department

$4.2 \pm 6.1$

Upper GI

$139(17)$

Lower GI

$221(27)$

Hepatobiliary-pancreas

$180(22)$

Vascular

149 (18.2)

Trauma

87 (10.6)

Miscellaneous

$42(5.1)$

\section{Sonographic findings $(\mathrm{N}=4,872)$}

Chest

Pleural effusion

$1,203(24.7)$

Pneumothorax

$10(0.2)$

Pulmonary edema

465 (9.5)

Hepatobiliary (liver, GB, pancreas, spleen)

Fluid collection $429(8.8)$

Abdomen and pelvis

Mass or hematoma

97 (2)

Cholecystitis or stone, polyp

$395(8.1)$

Fluid collection

925 (19)

Mass or hematoma

$243(5)$

Hydronephrosis

49 (1)

IVC dilatation

Normal

$1765(36.2)$

Collapsed

$1429(29.3)$

Dilated

$1352(27.8)$

Not checkable

331 (6.8)

IVC collapsibility index*

$19.6 \pm 13.8$

* An experience group is defined as the participants who had performed bedside ultrasound on at least five times in clinical setting prior to study enrollment. A participant in which the average number of experiences of bedside ultrasound before the study enrollment was less than five was defined as the inexperienced group.

** The IVC collapsibility index (IVCCI=[(IVCe-IVCi)/IVCe]×100\%) was calculated as the IVC provided respiratory variation. The inspiratory (IVCi) and respiratory (IVCe) diameters of the IVC were detected by measuring the vein lumen at 1 respiratory cycle.

Based on the proficiency and OSAUS scores, there were a significant increase in the participants' confidence level measured after the training course across all items $(P<0.001)$ compared with the level measured before the training (Figure 4$)$. In subgroup analysis junior residents (PGY1 and PGY2) showed less 
improvement in the post-course score, whereas PGY4 showed significant improvement in every element measured $(P<0.001)$ as shown in Table 2 .

Table 2

Scores for each item in terms of comprehension and confidence in the technique from pre- and post-course evaluation for each trainee

\begin{tabular}{|c|c|c|c|c|c|c|c|c|c|c|c|c|c|}
\hline \multirow{2}{*}{$\begin{array}{l}\text { Trainee \& } \\
\text { training period } \\
\text { (week) }\end{array}$} & \multicolumn{3}{|c|}{$P G Y * 1(n=11)$} & \multicolumn{3}{|c|}{ PGY $2(n=9)$} & \multicolumn{3}{|c|}{ PGY $3(n=9)$} & \multicolumn{3}{|c|}{ PGY $4(n=15)$} & \multirow{2}{*}{$\begin{array}{l}\text { Tota } \\
\text { Pre } \\
\text { cour }\end{array}$} \\
\hline & $\begin{array}{l}\text { Pre- } \\
\text { course }\end{array}$ & $\begin{array}{l}\text { Post- } \\
\text { course }\end{array}$ & $\begin{array}{l}\mathrm{P} \\
\text { value }\end{array}$ & $\begin{array}{l}\text { Pre- } \\
\text { course }\end{array}$ & $\begin{array}{l}\text { Post- } \\
\text { course }\end{array}$ & $\begin{array}{l}\mathrm{P} \\
\text { value }\end{array}$ & $\begin{array}{l}\text { Pre- } \\
\text { course }\end{array}$ & $\begin{array}{l}\text { Post- } \\
\text { course }\end{array}$ & $\begin{array}{l}\mathrm{P} \\
\text { value }\end{array}$ & $\begin{array}{l}\text { Pre- } \\
\text { course }\end{array}$ & $\begin{array}{l}\text { Post- } \\
\text { course }\end{array}$ & $\begin{array}{l}\mathrm{P} \\
\text { value }\end{array}$ & \\
\hline \multicolumn{14}{|l|}{$\begin{array}{l}\text { Proficiency in } \\
\text { manipulation }\end{array}$} \\
\hline $\begin{array}{l}\text { Lung } \\
\text { parenchyme }\end{array}$ & $\begin{array}{l}1.9 \pm \\
0.7\end{array}$ & $\begin{array}{l}3.8 \pm \\
0.5\end{array}$ & 0.001 & $\begin{array}{l}2.2 \pm \\
0.8\end{array}$ & $\begin{array}{l}3.8 \pm \\
0.5\end{array}$ & 0.006 & $\begin{array}{l}2.4 \pm \\
0.5\end{array}$ & $\begin{array}{l}4.2 \pm \\
0.8\end{array}$ & 0.002 & $\begin{array}{l}2.1 \pm \\
0.7\end{array}$ & $4 \pm 1.1$ & <..001 & $\begin{array}{l}2.1 \pm \\
0.7\end{array}$ \\
\hline $\begin{array}{l}\text { Pleural } \\
\text { effusion }\end{array}$ & $\begin{array}{l}1.6 \pm \\
0.8\end{array}$ & $2 \pm 0.8$ & 0.413 & $2 \pm 0.6$ & $3.5 \pm 1$ & 0.019 & $\begin{array}{l}2.8 \pm \\
0.8\end{array}$ & $\begin{array}{l}3.7 \pm \\
0.5\end{array}$ & 0.064 & $\begin{array}{l}2.9 \pm \\
0.8\end{array}$ & $\begin{array}{l}4.4 \pm \\
0.7\end{array}$ & $<.001$ & $\begin{array}{l}2.6 \pm \\
0.9\end{array}$ \\
\hline Bowel & $\begin{array}{l}1.9 \pm \\
0.4\end{array}$ & $\begin{array}{l}2.3 \pm \\
0.5\end{array}$ & 0.172 & $2 \pm 0.6$ & $3 \pm 0.8$ & 0.060 & $\begin{array}{l}1.4 \pm \\
0.5\end{array}$ & $\begin{array}{l}3.3 \pm \\
0.5\end{array}$ & $\dot{0} 001$ & $\begin{array}{l}2.8 \pm \\
0.7\end{array}$ & $\begin{array}{l}4.2 \pm \\
0.7\end{array}$ & $<.001$ & $\begin{array}{l}2.5 \pm \\
0.9\end{array}$ \\
\hline $\begin{array}{l}\text { Peritoneal } \\
\text { cavity }\end{array}$ & $\begin{array}{l}1.3 \pm \\
0.5\end{array}$ & $\begin{array}{l}2.3 \\
\pm .5\end{array}$ & 0.012 & $\begin{array}{l}1.7 \pm \\
0.5\end{array}$ & $\begin{array}{l}2.8 \pm \\
0.5\end{array}$ & 0.011 & $\begin{array}{l}2.4 \pm \\
0.9\end{array}$ & $\begin{array}{l}3.8 \pm \\
0.4\end{array}$ & 0.006 & $\begin{array}{l}2.9 \pm \\
0.4\end{array}$ & $\begin{array}{l}4.4 \pm \\
0.6\end{array}$ & $\dot{0} 001$ & $\begin{array}{l}2.5 \pm \\
0.8\end{array}$ \\
\hline Hepatobiliary & $2 \pm 0.8$ & $3 \pm 0.8$ & 0.082 & $\begin{array}{l}2.3 \pm \\
0.8\end{array}$ & $\begin{array}{l}3.5 \pm \\
0.6\end{array}$ & 0.040 & $3 \pm 0.7$ & $\begin{array}{l}3.3 \pm \\
0.8\end{array}$ & 0.493 & $\begin{array}{l}2.9 \pm \\
0.8\end{array}$ & $\begin{array}{l}4.2 \pm \\
0.7\end{array}$ & $<.001$ & $\begin{array}{l}2.7 \pm \\
0.8\end{array}$ \\
\hline IJV & $3 \pm 0.8$ & $\begin{array}{l}3.8 \pm \\
0.5\end{array}$ & 0.134 & $\begin{array}{l}2.8 \pm \\
1.3\end{array}$ & $\begin{array}{l}3.8 \pm \\
0.5\end{array}$ & 0.231 & $\begin{array}{l}3.8 \pm \\
0.8\end{array}$ & $\begin{array}{l}3.5 \pm \\
0.5\end{array}$ & 0.492 & $\begin{array}{l}4.3 \pm \\
0.5\end{array}$ & $\begin{array}{l}4.8 \pm \\
0.5\end{array}$ & $\dot{0} 001$ & $\begin{array}{l}3.9 \pm \\
0.9\end{array}$ \\
\hline IVC & $\begin{array}{l}2.3 \pm \\
0.8\end{array}$ & $3 \pm 1.4$ & 0.294 & $\begin{array}{l}1.8 \pm \\
0.8\end{array}$ & $\begin{array}{l}3.3 \pm \\
0.5\end{array}$ & 0.011 & $\begin{array}{l}3.2 \pm \\
0.4\end{array}$ & $\begin{array}{l}3.7 \pm \\
0.8\end{array}$ & 0.285 & $\begin{array}{l}3.8 \pm \\
0.8\end{array}$ & $\begin{array}{l}4.7 \pm \\
0.6\end{array}$ & $<0.001$ & $\begin{array}{l}3.2 \pm \\
1.1\end{array}$ \\
\hline \multicolumn{14}{|l|}{ OSAUS $^{\star * *}$} \\
\hline $\begin{array}{l}\text { Applied } \\
\text { knowledge }\end{array}$ & $\begin{array}{l}2.3 \pm \\
0.5\end{array}$ & 3 & 0.008 & $\begin{array}{l}2.8 \pm \\
0.4\end{array}$ & $3.3 \pm 1$ & 0.363 & 3 & $\begin{array}{l}3.3 \pm \\
0.5\end{array}$ & 0.175 & $\begin{array}{l}3.1 \pm \\
0.7\end{array}$ & $\begin{array}{l}4.6 \pm \\
0.6\end{array}$ & $\dot{0.001}$ & $\begin{array}{l}2.9 \pm \\
0.7\end{array}$ \\
\hline $\begin{array}{l}\text { Image } \\
\text { optimization }\end{array}$ & $\begin{array}{l}1.6 \pm \\
0.5\end{array}$ & $\begin{array}{l}3.3 \pm \\
0.5\end{array}$ & 0.001 & $\begin{array}{l}2.5 \pm \\
0.5\end{array}$ & $3.3 \pm 1$ & 0.150 & 3 & 4 & - & $\begin{array}{l}2.8 \pm \\
0.6\end{array}$ & $4 \pm 0.8$ & $<0.001$ & $\begin{array}{l}2.6 \\
\pm .7\end{array}$ \\
\hline $\begin{array}{l}\text { Systemic } \\
\text { examination }\end{array}$ & $\begin{array}{l}2.3 \pm \\
0.8\end{array}$ & $\begin{array}{l}2.5 \pm \\
0.6\end{array}$ & 0.638 & $\begin{array}{l}2.7 \pm \\
0.5\end{array}$ & $\begin{array}{l}3.3 \pm \\
0.5\end{array}$ & 0.115 & 2 & $\begin{array}{l}3.7 \pm \\
0.5\end{array}$ & 0.001 & $\begin{array}{l}2.8 \pm \\
0.4\end{array}$ & $\begin{array}{l}4.2 \pm \\
0.7\end{array}$ & < 0.001 & $\begin{array}{l}2.6 \pm \\
0.5\end{array}$ \\
\hline $\begin{array}{l}\text { Interpretation } \\
\text { of images }\end{array}$ & $\begin{array}{l}2.3 \pm \\
0.8\end{array}$ & $\begin{array}{l}2.5 \pm \\
0.6\end{array}$ & 0.638 & $\begin{array}{l}2.3 \pm \\
0.5\end{array}$ & $2.8 \pm 1$ & 0.393 & $\begin{array}{l}2.4 \pm \\
0.5\end{array}$ & $\begin{array}{l}3.7 \pm \\
0.5\end{array}$ & 0.003 & $\begin{array}{l}3.2 \pm \\
0.5\end{array}$ & $\begin{array}{l}4.2 \pm \\
0.6\end{array}$ & <. 001 & $\begin{array}{l}2.9 \pm \\
0.7\end{array}$ \\
\hline $\begin{array}{l}\text { Documentation } \\
\text { of examination }\end{array}$ & $\begin{array}{l}1.9 \pm \\
0.4\end{array}$ & $\begin{array}{l}2.3 \pm \\
0.5\end{array}$ & 0.172 & $\begin{array}{l}2.2 \pm \\
0.8\end{array}$ & $2.5 \pm 1$ & 0.562 & $\begin{array}{l}2.4 \pm \\
0.5\end{array}$ & $\begin{array}{l}3.7 \pm \\
0.5\end{array}$ & 0.003 & $\begin{array}{l}3.2 \pm \\
0.7\end{array}$ & $\begin{array}{l}4.2 \pm \\
0.6\end{array}$ & <.001 & $\begin{array}{l}2.8 \pm \\
0.8\end{array}$ \\
\hline
\end{tabular}

*PGY (Postgraduate year)

Additionally, the maximum improvement in all elements was observed in the proficiency score of lung parenchyma (pre-course score $=2.1 \pm 0.7$, post-course score $=4 \pm 1$ ). Table 3 presents the comparative analysis of mean differences before and after the training course of each PGY group. The degree of improvement between the PGY groups showed significant differences in proficiency of manipulation and OSAUS scores after the training, except for the proficiency in peritoneal cavity. The post hoc test was performed to compare the results between the groups classified according to the residency year. In the comparison based on residency year, PGY 2 showed the most significant improvement. PGY 2 showed significant differences in five of seven elements involving proficiency in manipulation and three of five elements in OSAUS score, whereas PGY 1 showed differences in a single element. The largest mean differences were seen in the proficiency score of bowel between PGY 3 and PGY 1 , (mean difference $=2.5, P<0.001$ ) followed by PGY 2.0 and PGY 1 (mean difference $=2, P<0.001)$. The post hoc test showed no differences in the proficiency scores of peritoneal cavity and IVC between any of the PGY groups. 
Table 3

Differences of pre- and post-course evaluation scores in terms of comprehension and confidence in the technique for each group

\begin{tabular}{|c|c|c|c|c|c|c|c|c|c|c|}
\hline & & \multirow{2}{*}{$\begin{array}{l}P G Y^{*} 1 \\
(n= \\
11)\end{array}$} & \multirow{2}{*}{$\begin{array}{l}\text { PGY } 2 \\
(n=9)\end{array}$} & \multirow{2}{*}{$\begin{array}{l}\text { PGY } 3 \\
(n=9)\end{array}$} & \multirow{2}{*}{$\begin{array}{l}\text { PGY } 4 \\
(n= \\
15)\end{array}$} & \multirow{2}{*}{$\begin{array}{l}\text { Total } \\
(n= \\
44)\end{array}$} & \multirow{2}{*}{$\begin{array}{l}p- \\
\text { value }\end{array}$} & \multicolumn{3}{|c|}{ Post-hoc Analysis ${ }^{\dagger}$} \\
\hline & & & & & & & & Comparisons & $\begin{array}{l}\text { Mean } \\
\text { difference }\end{array}$ & $\begin{array}{l}\mathrm{p}- \\
\text { value }\end{array}$ \\
\hline \multirow[t]{11}{*}{$\begin{array}{l}\text { Proficiency in } \\
\text { manipulation }\end{array}$} & Lung parenchyme & $\begin{array}{l}1.7 \pm \\
1.2\end{array}$ & $\begin{array}{l}2.5 \pm \\
0.7\end{array}$ & $\begin{array}{l}1.5 \pm \\
2.1\end{array}$ & $1.8 \pm 1$ & $\begin{array}{l}1.8 \pm \\
1.1\end{array}$ & 0.014 & $\begin{array}{l}\text { PGY } 2 \text { - PGY } \\
3\end{array}$ & 1 & 0.033 \\
\hline & Pleural effusion & $\begin{array}{l}0.7 \pm \\
0.6\end{array}$ & $\begin{array}{l}2.5 \pm \\
0.7\end{array}$ & $\begin{array}{l}0.5 \pm \\
0.7\end{array}$ & $2 \pm 1.4$ & $\begin{array}{l}1.5 \pm \\
1.2\end{array}$ & $\dot{0} 0001$ & $\begin{array}{l}\text { PGY } 2 \text { - PGY } \\
1\end{array}$ & 1.833 & $\hat{0} .001$ \\
\hline & & & & & & & & $\begin{array}{l}\text { PGY } 2 \text { - PGY } \\
3\end{array}$ & 2 & ¿.001 \\
\hline & Bowel & $0 \pm 1.7$ & $2 \pm 1.4$ & $\begin{array}{l}2.5 \pm \\
0.7\end{array}$ & $1.5 \pm 1$ & $\begin{array}{l}1.4 \pm \\
1.4\end{array}$ & $<.001$ & $\begin{array}{l}\text { PGY } 2 \text { - PGY } \\
1\end{array}$ & 2 & $<.001$ \\
\hline & & & & & & & & $\begin{array}{l}\text { PGY } 3-P G Y \\
1\end{array}$ & 2.5 & <. 001 \\
\hline & Peritoneal cavity & $\begin{array}{l}1.3 \pm \\
0.6\end{array}$ & 2 & $\begin{array}{l}1.5 \pm \\
2.1\end{array}$ & $\begin{array}{l}1.5 \pm \\
1.3\end{array}$ & $1.5 \pm 1$ & 0.163 & - & & \\
\hline & Hepatobiliary & $1 \pm 1$ & 2 & $\begin{array}{l}1.5 \pm \\
0.7\end{array}$ & $1.3 \pm 1$ & $\begin{array}{l}1.4 \pm \\
0.8\end{array}$ & $<0.001$ & $\begin{array}{l}\text { PGY } 2 \text { - PGY } \\
1\end{array}$ & 1 & ¿.001 \\
\hline & & & & & & & & $\begin{array}{l}\text { PGY } 2 \text { - PGY } \\
4\end{array}$ & 0.750 & 0.005 \\
\hline & IJV & $\begin{array}{l}0.3 \pm \\
1.5\end{array}$ & $\begin{array}{l}1.5 \pm \\
0.7\end{array}$ & $0 \pm 1.4$ & $\begin{array}{l}0.5 \pm \\
0.6\end{array}$ & $0.5 \pm 1$ & $<.001$ & $\begin{array}{l}\text { PGY } 2-P G Y \\
1\end{array}$ & 1.167 & 0.001 \\
\hline & & & & & & & & $\begin{array}{l}\text { PGY } 2 \text { - PGY } \\
3\end{array}$ & 1.5 & <. 001 \\
\hline & IVC & $1 \pm 1.7$ & $2 \pm 1.4$ & $\begin{array}{l}1.5 \pm \\
0.7\end{array}$ & $1 \pm 1.8$ & $\begin{array}{l}1.3 \pm \\
1.4\end{array}$ & 0.040 & - & & \\
\hline \multirow[t]{8}{*}{ OSAUS ${ }^{* *}$} & Applied knowledge & 1 & 1 & $\begin{array}{l}0.5 \pm \\
0.7\end{array}$ & $\begin{array}{l}1.5 \pm \\
1.3\end{array}$ & $\begin{array}{l}1.1 \pm \\
0.8\end{array}$ & $<0.001$ & $\begin{array}{l}\text { PGY } 4-P G Y \\
3\end{array}$ & 1 & $\dot{0} 0.001$ \\
\hline & Image optimization & $\begin{array}{l}1.7 \pm \\
1.2\end{array}$ & $\begin{array}{l}1.5 \pm \\
0.7\end{array}$ & 1 & $1.5 \pm 1$ & $\begin{array}{l}1.5 \pm \\
0.8\end{array}$ & 0.040 & $\begin{array}{l}\text { PGY } 1 \text { - PGY } \\
3\end{array}$ & 0.667 & 0.048 \\
\hline & Systemic examination & $\begin{array}{l}0.3 \pm \\
0.6\end{array}$ & $\begin{array}{l}1.5 \pm \\
0.7\end{array}$ & 2 & $1.5 \pm 1$ & $\begin{array}{l}1.3 \pm \\
0.9\end{array}$ & $\dot{0} 0.001$ & $\begin{array}{l}\text { PGY } 2 \text { - PGY } \\
1\end{array}$ & 1.167 & <. 001 \\
\hline & & & & & & & & $\begin{array}{l}\text { PGY } 3-P G Y \\
1\end{array}$ & 1.667 & $<.001$ \\
\hline & Interpretation of images & $\begin{array}{l}0.3 \pm \\
0.6\end{array}$ & $\begin{array}{l}1.5 \pm \\
0.7\end{array}$ & 2 & $\begin{array}{l}1.5 \pm \\
0.6\end{array}$ & $\begin{array}{l}1.3 \pm \\
0.8\end{array}$ & $\hat{0} .001$ & $\begin{array}{l}\text { PGY } 2 \text { - PGY } \\
1\end{array}$ & 1.2 & ¿. 001 \\
\hline & & & & & & & & $\begin{array}{l}\text { PGY } 3-P G Y \\
2\end{array}$ & 0.5 & 0.015 \\
\hline & $\begin{array}{l}\text { Documentation of } \\
\text { examination }\end{array}$ & 1 & $\begin{array}{l}1.5 \pm \\
0.7\end{array}$ & 1 & $\begin{array}{l}1.8 \pm \\
0.5\end{array}$ & $\begin{array}{l}1.4 \pm \\
0.5\end{array}$ & $\hat{0} .001$ & $\begin{array}{l}\text { PGY } 2 \text { - PGY } \\
1,3\end{array}$ & 0.5 & 0.005 \\
\hline & & & & & & & & $\begin{array}{l}\text { PGY } 4 \text { - PGY } \\
1,3\end{array}$ & 0.8 & $<.001$ \\
\hline
\end{tabular}

*PGY (Postgraduate year)

As shown in Table 4, we also performed a subgroup analysis and compared experienced and inexperienced residents to determine if a previous exposure to bedside sonogram affected the training results. Both groups demonstrated significantly higher confidence after the completion of course in all areas of evaluation. Comparing the advances between the two groups, only proficiency in manipulation score involving pleural effusion showed differences in the degree of improvement (experienced 2.9 versus 4.4 , inexperienced 2.1 versus 3.6. $P<0.001$ ) In OSAUS score, experienced residents showed better improvement except in image optimization where both groups showed improvement $(P=0.666)$. Experienced residents achieved significantly higher postcourse confidence than inexperienced residents and the extent of the increment was greater. 
Table 4

Differences of pre- and post-course evaluation in terms of Comprehension \& confidence in the technique ${ }^{\star}$ of Experienced and Inexperienced residents. (Average of mean difference)

\begin{tabular}{|c|c|c|c|c|c|c|c|}
\hline & \multicolumn{3}{|c|}{ Experienced $(n=16)$} & \multicolumn{3}{|c|}{ Inexperienced $(\mathrm{n}=28)$} & \multirow[t]{2}{*}{$p$-value } \\
\hline & Pre-course & Post-course & p-value & Pre-course & Post-course & p-value & \\
\hline \multicolumn{8}{|l|}{ Proficiency in manipulation } \\
\hline Lung parenchyme & $2.1 \pm 0.7$ & $4 \pm 1.1$ & $<0.001$ & $2.1 \pm 0.7$ & $4 \pm 0.7$ & $<0.001$ & 0.594 \\
\hline Pleural effusion & $2.9 \pm 0.8$ & $4.4 \pm 0.7$ & $<0.001$ & $2.1 \pm 0.9$ & $3.6 \pm 0.7$ & $<0.001$ & $<0.001$ \\
\hline Bowel & $2.8 \pm 0.7$ & $4.2 \pm .7$ & $<0.001$ & $1.8 \pm 0.9$ & $3.2 \pm 0.6$ & $<0.001$ & 0.390 \\
\hline Peritoneal cavity & $2.9 \pm 0.4$ & $4.4 \pm 0.6$ & $<0.001$ & $1.7 \pm 0.8$ & $3.4 \pm 0.7$ & $<0.001$ & 0.748 \\
\hline Hepatobiliary & $2.9 \pm 0.8$ & $4.2 \pm 0.7$ & $<0.001$ & $2.4 \pm 0.9$ & $3.4 \pm 0.7$ & 0.004 & 0.272 \\
\hline IJV & $4.3 \pm 0.5$ & $4.8 \pm 0.5$ & $<0.001$ & $3.2 \pm 1$ & $3.6 \pm 0.5$ & 0.231 & 0.679 \\
\hline IVC & $3.8 \pm 0.8$ & $4.7 \pm 0.6$ & $<0.001$ & $2.4 \pm 0.9$ & $3.5 \pm 0.7$ & 0.002 & 0.167 \\
\hline \multicolumn{8}{|l|}{ OSAUS ${ }^{* *}$} \\
\hline Applied knowledge & $3.1 \pm 0.7$ & $4.6 \pm 0.6$ & $<0.001$ & $2.7 \pm 0.5$ & $3.3 \pm 0.7$ & 0.008 & 0.002 \\
\hline Image optimization & $2.8 \pm 0.6$ & $4 \pm 0.8$ & $<0.001$ & $2.3 \pm 0.8$ & $3.7 \pm 0.7$ & $<0.001$ & 0.666 \\
\hline Systemic examination & $2.8 \pm 0.4$ & $4.2 \pm 0.7$ & $<0.001$ & $2.3 \pm 0.6$ & $3.5 \pm 0.5$ & $<0.001$ & 0.048 \\
\hline Interpretation of images & $3.2 \pm 0.5$ & $4.2 \pm 0.6$ & $<0.001$ & $2.3 \pm 0.6$ & $3.3 \pm 0.8$ & 0.001 & 0.010 \\
\hline Documentation of examination & $3.2 \pm 0.7$ & $4.2 \pm 0.6$ & $<0.001$ & $2.1 \pm 0.6$ & $3.2 \pm 0.9$ & 0.001 & $<0.001$ \\
\hline
\end{tabular}

* Level of confidence rated on a 5-point Likert scale ( $1=$ not confident at all $/ 3=$ neutral $/ 5=\overline{v e r y ~ c o n f i d e n t) ~}$

**OSAUS (The Objective Structured Assessment of Ultrasound Skills) on a 5-point Likert scale $(1=$ not confident at all $/ 3=$ neutral $/ 5=$ very confident $)$

\section{Discussion}

Based on our results, surgical residents showed a significant improvement in ultrasound basics and focused assessment of bedside sonography for critical ill patients after participation in our training curriculum. Additionally, the senior residents (PGY 3 and 4) showed a significant increase in scores in more areas of evaluation compared with junior residents. All residents showed significant improvement regardless of prior experience with performing bedside sonography. Ultrasound is generally used in diagnostic and procedural investigation, and in routine clinical practice owing to its efficacy and safety [2]. Technological advances have led to the use of high-performance ultrasound, which is increasingly compact and portable. Thus, bedside ultrasonography can enable acquisition of real-time images by the clinician. It facilitates surgical diagnosis and procedures with improved success rate and decreased complications [6]. Especially in trauma or critically ill patients, the usage of bedside ultrasound can enable the identification of the etiology of certain conditions such as hypotensive shock or respiratory failure. Bedside sonography, such as e-FAST examination, has a sensitivity of $73 \sim 99 \%$, a specificity of $94 \sim 98 \%$, and an overall accuracy of $90 \sim 98 \%$ for intra-abdominal injury in trauma, and a sensitivity of $78.6 \%$ and a specificity of $98.4 \%$ for detecting pneumothorax [6, 18]. The need for additional diagnostic tests such as CT scans can be reduced, thus shortening the time that takes to implement appropriate intervention [7]. From an educational viewpoint, even inexperienced learners can perform bedside sonographic examination easily, with proficiency and accuracy comparable to that of a radiologist $[19,20]$. However, despite these advantages, a formal training curriculum in ultrasound is still lacking in many surgical residencies. Our results showed that most residents were unfamiliar with the use of ultrasound or bedside ultrasound evaluation before. But, after the completion of ultrasound training program, they could achieve a significant improvement in their knowledge and confidence compared to the pre-course score levels. Authors expect that a well-organized and systematic program in bedside ultrasound training could ultimately help the residents, who conduct initial resuscitation in critically ill surgical patients.

Additionally, our results shown in Table 2 suggest that the pre-course scores in proficiency and OSAUS were similarly low regardless of the year of training. However, PGY 4 groups showed improvement in every area of evaluation while only few areas were improved in the PGY1 group, probably because senior residents have more experience in clinical settings involving relatively diverse surgery. Prior knowledge of the key elements of the altered anatomy after the surgery or specific findings related to the clinical condition can facilitate evaluation of the subjects more intensively during the ultrasound examination. It will enable the educational and learning outcomes during the training. Therefore, we expect that the trainee's prior knowledge and understanding of patients' anatomy or surgery such as in the case of senior residents, the better is the educational impact of our training program.

Noteworthy, PGY 2 residents exhibited the most significant findings during the post-training evaluation in our results. In Table 3, when comparing responses of our program by training years, significant differences existed between PGY groups. Except in applied knowledge and image optimization, PGY 2 residents showed a higher mean difference in most elements than the other groups. However, PGY 4 had the highest post-course scores, but the pre-course score was higher than in PGY 2 participants, which explains why our senior residents did not show a higher mean difference than PGY 2 . PGY 1 residents exhibited the lowest responses after the training program, which might be attributed to limited understanding of anatomical structure and ultrasound of novice trainee. Therefore, we expect that our training program would be most suitable and effective for surgical residents with at least basic knowledge of surgical anatomy, such as in the case of PGY 2. 
When compared the scores according to previous ultrasound experience before training, residents with prior experience showed higher pre-course score than those of residents without experience of sonography. But after completing the training course, there was a meaningful improvement in scores in both groups. These results suggest that our ultrasound training program can help trainees with less experience in ultrasound manipulations acquire ultrasound skills and clinical interpretation more effectively by providing dense hands-on opportunities for short periods of time. Our training program is not only useful to novice residents, but also enhances the understanding and confidence levels of non-beginners with little experience.

Despite these interesting findings, the current study had inherent limitations that require attention. First, this study has small number of trainees and involves a single institution. Consequently, our training results may not be generalized to other institutions. To establish the reliability and reproducibility of our results and their applicability to other training hospitals, a large-scale study with a large number of trainees across different training hospitals is needed. Second, this study did not test the accuracy of sonography or resident's ability to perform and interpret in the clinical setting after the training. In order to use ultrasound in medical practice, the efficacy of training program on clinical performance should be evaluated [21, 22]. Third, unlike other ultrasound training programs, we did not use simulator or healthy human model. In our program, we performed ultrasound in actual patients who underwent surgeries or patients who were in an unstable condition. Therefore, it was not easy to identify every important structure each time or visualize a normal image of uninjured organ. Meanwhile, our results provide insight into lack of ultrasound skills or education in a surgical resident for appropriate ultrasound training program to enhance the resident's ultrasound skills and confidence, effectively. Authors believe that a prospective multi-center trial with large number of participants should be conducted in the near future to corroborate our study results.

In conclusion, our short and intense bedside ultrasound training program improves the confidence and skills of all surgical residents regardless of the resident's PGY or prior experiences. Given the diversity of applications of bedside ultrasound in surgical medicine, we believe that our training curriculum in bedside sonography for critical patients would be beneficial for all surgical residencies.

\section{Abbreviations}

\section{OSAUS}

the objective structured assessment of ultrasound skill

ICU

Intensive care unit

ACS

The American College of Surgeons

KSS

the Korean Surgical Society

PGY

post-graduate year

e-FAST

extended-Focused Assessment with Sonography for Trauma

IVC

inferior vena cava

\section{Declarations}

\section{Ethics approval and consent to participate}

This study was approved by the Institutional Review Board of the Ethics Committee of the College of Medicine, The Catholic University of Korea (KC20QISI0723). The written informed consent from the participants were obtained, and the procedures were in compliance with Helsinki Declaration.

\section{Consent for publication}

Written consent from the participants were obtained.

\section{Availability of data and materials}

The dataset supporting the conclusions of this article is included within the article (and its additional file(s)), and can be obtained from the corresponding author on a reasonable request.

\section{Competing interests}

The authors report no conflicts of interest.

\section{Funding}

None.

\section{Authors' contributions}


$\mathrm{KMI}$ and EYK designed and conducted the study. KMI and EYK collected and analyzed the data. KMI drafted the manuscript and EYK made critical revision of the manuscript. All authors reviewed and approved the final version of the manuscript, and agreed to be accountable for all aspects of the work in ensuring the questions related to the accuracy or integrity of any part of the work are appropriately investigated and resolved.

\section{Acknowledgements}

The authors deeply appreciate the surgical residents and supervisors at Seoul St. Mary's Hospital who participated in the training course. The authors received no financial support for the research, authorship, and/or publication of this article.

\section{References}

1. Lee JH, Kang B, Kwon H, Kim S, Kim JY, Lee JH, Jung JY, Ha Y-R: The Experience of Emergency Pediatric Ultrasound Education Course. Pediatr Emerg Med J 2014, 1(1):34-41.

2. Beggs AD, Thomas PR: Point of use ultrasound by general surgeons: review of the literature and suggestions for future practice. International Journal of Surgery 2013, 11(1):12-17.

3. Ghane MR, Gharib M, Ebrahimi A, Saeedi M, Akbari-Kamrani M, Rezaee M, Rasouli H: Accuracy of early rapid ultrasound in shock (RUSH) examination performed by emergency physician for diagnosis of shock etiology in critically ill patients. Journal of emergencies, trauma, and shock 2015, 8(1):5.

4. Nassour I, Spalding MC, Hynan LS, Gardner AK, Williams BH: The surgeon-performed ultrasound: a curriculum to improve residents' basic ultrasound knowledge. The Journal of surgical research 2017, 213:51-59.

5. Carver TW: Ultrasound Training in Surgical Critical Care Fellowship: A Survey of Program Directors. Journal of surgical education 2018, 75(5):1250-1255.

6. Moore CL, Copel JA: Point-of-Care Ultrasonography. New England Journal of Medicine 2011, 364(8):749-757.

7. Cevik AA, Noureldin A, El Zubeir M, Abu-Zidan FM: Assessment of EFAST training for final year medical students in emergency medicine clerkship. Turkish Journal of Emergency Medicine 2018, 18(3):100-104.

8. Helling TS, Wilson J, Augustosky K: The utility of focused abdominal ultrasound in blunt abdominal trauma: a reappraisal. American journal of surgery 2007, 194(6):728-732; discussion 732 - 723.

9. Fuchs L, Chronaki CE, Park S, Novack V, Baumfeld Y, Scott D, McLennan S, Talmor D, Celi L: ICU admission characteristics and mortality rates among elderly and very elderly patients. Intensive care medicine 2012, 38(10):1654-1661.

10. Kim JS, Cho YS, Kim YS, Ha YR, Kang BS, Chung YS, Park YS, An JH, Do HH, Hong HP: Development of an Emergency Abdominal Ultrasound Course in Korea: 1-Year Experience. In: Taehan Ǔnggup Ǔihakhoe chi. vol. 21; 2010: 382.

11. Martin JA, Regehr G, Reznick R, MacRae H, Murnaghan J, Hutchison C, Brown M: Objective structured assessment of technical skill (OSATS) for surgical residents. The British journal of surgery 1997, 84(2):273-278.

12. Crouch AK, Dawson M, Long D, Allred D, Madsen T: Perceived confidence in the FAST exam before and after an educational intervention in a developing country. International journal of emergency medicine 2010, 3(1):49-52.

13. Pulijala Y, Ma M, Pears M, Peebles D, Ayoub A: Effectiveness of Immersive Virtual Reality in Surgical Training-A Randomized Control Trial. Journal of Oral and Maxillofacial Surgery 2018, 76(5):1065-1072.

14. Fonseca AL, Reddy V, Yoo PS, Gusberg RJ, Longo WE: Senior Surgical Resident Confidence in Performing Flexible Endoscopy: What Can We Do Differently? Journal of Surgical Education 2016, 73(2):311-316.

15. Happel CS, Lease MA, Nishisaki A, Braga MS: Evaluating simulation education via electronic surveys immediately following live critical events: a pilot study. Hospital pediatrics 2015, 5(2):96-100.

16. Schroll R, Smith A, Martin MS, Zeoli T, Hoof M, Duchesne J, Greiffenstein P, Avegno J: Stop the Bleed Training: Rescuer Skills, Knowledge, and Attitudes of Hemorrhage Control Techniques. The Journal of surgical research 2020, 245:636-642.

17. Tolsgaard MG, Todsen T, Sorensen JL, Ringsted C, Lorentzen T, Ottesen B, Tabor A: International multispecialty consensus on how to evaluate ultrasound competence: a Delphi consensus survey. PloS one 2013, 8(2):e57687.

18. Alrajab S, Youssef AM, Akkus NI, Caldito G: Pleural ultrasonography versus chest radiography for the diagnosis of pneumothorax: review of the literature and meta-analysis. Critical Care 2013, 17(5):1-8.

19. Williams R, Windsor A, Rosin RD, Mann DV, Crofton M: Ultrasound scanning of the acute abdomen by surgeons in training. Annals of the Royal College of Surgeons of England 1994, 76(4):228.

20. Nassour I, Spalding MC, Hynan LS, Gardner AK, Williams BH: The surgeon-performed ultrasound: a curriculum to improve residents' basic ultrasound knowledge. journal of surgical research 2017, 213:51-59.

21. Trajkovski T, Veillette C, Backstein D, Wadey VM, Kraemer B: Resident self-assessment of operative experience in primary total knee and total hip arthroplasty: Is it accurate? Canadian Journal of Surgery 2012, 55(4 Suppl 2):S153.

22. Arora S, Miskovic D, Hull L, Moorthy K, Aggarwal R, Johannsson H, Gautama S, Kneebone R, Sevdalis N: Self vs expert assessment of technical and nontechnical skills in high fidelity simulation. The American Journal of Surgery 2011, 202(4):500-506.

\section{Figures}




\section{Chest}

Pleural effusion

Pneumothorax

Pulmonary edema

Pneumonia

\section{Pericardium \& IVC}

Pericardial effusion

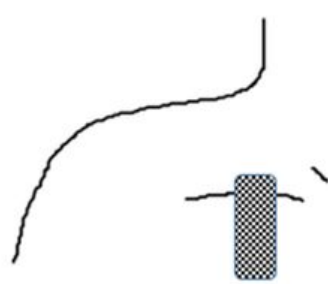

Assess IVC diameter

Assess IVC collapsibility index

\section{Liver}

Fluid collection at Morrison's pouch

Liver laceration

Liver abscess

Liver infarction

Cholecystitis

Cholelithiasis
Kidney

Kidney laceration

Hydronephrosis
O

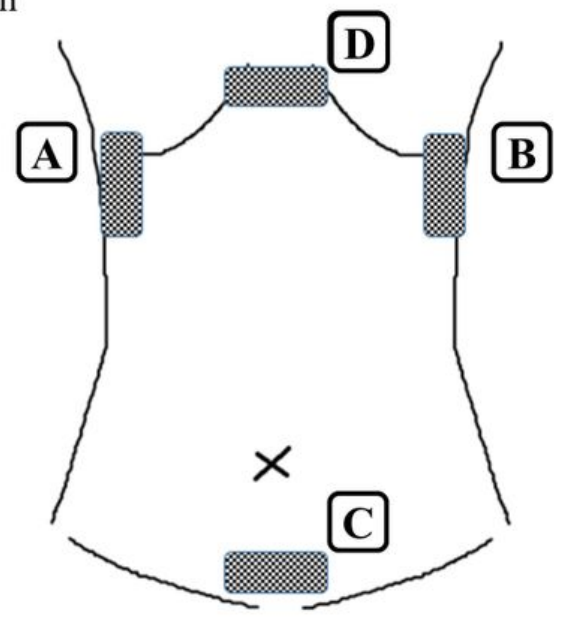

\section{Spleen}

Fluid collection at splenorenal angle Spleen laceration Spleen abscess Spleen infarction

\section{Pelvis}

Fluid collection at pouch of Douglas

Pneumothorax

Pulmonary edema

Pneumonia

\section{Figure 1}

The four abdominal views and chest view A. Morrison's pouch and the right diaphragm B. Spleno-renal angle and left diaphragm C. Pelvis in both longitudinal and transverse planes D. Pericardial E. Pleura (Bilaterally) 

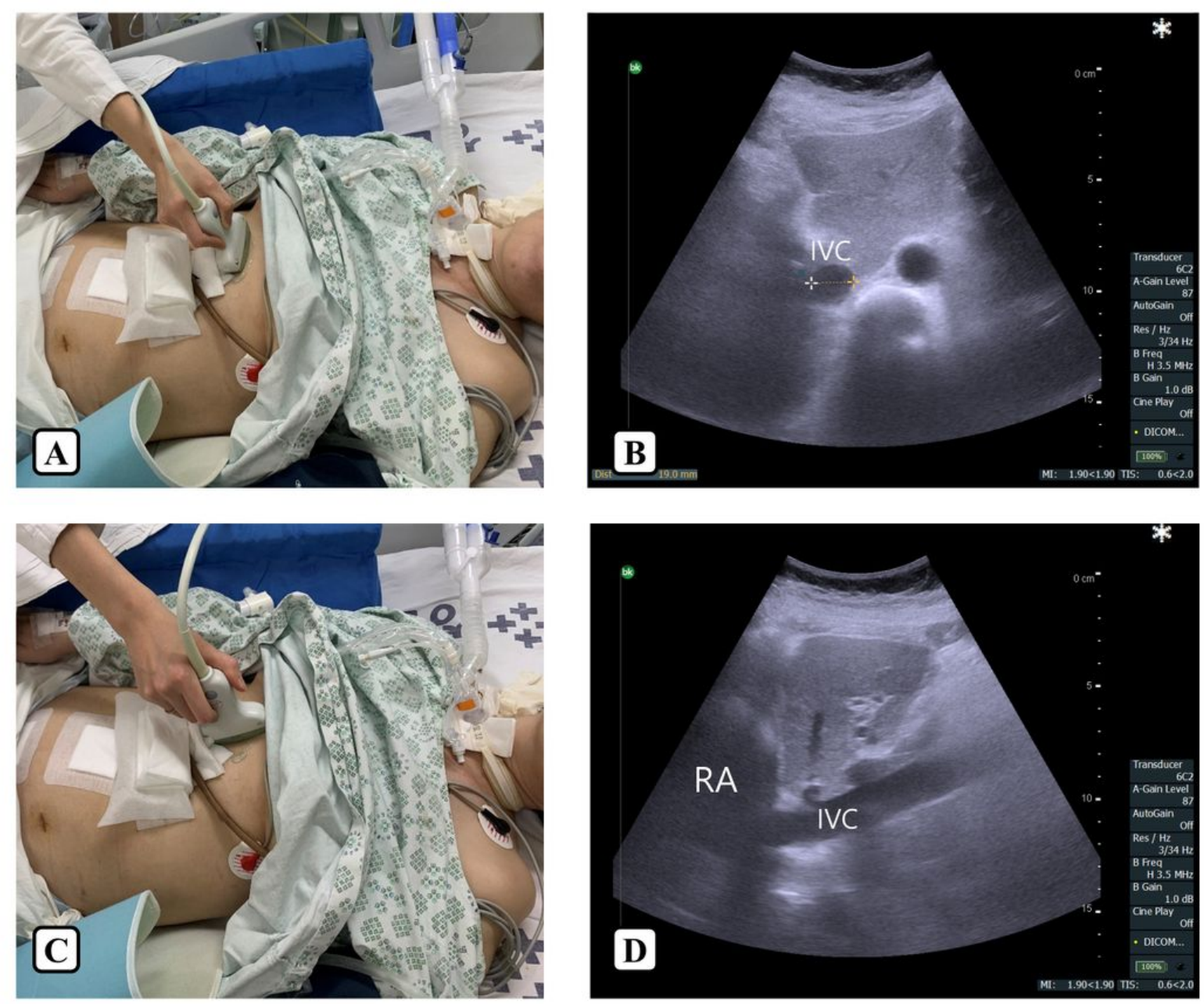

\section{Figure 2}

Participant evaluating the IVC diameter and its respiratory variation. A, B First IVC should be identified in a transverse plane, in a subxiphoid position perpendicular to the skin. C, D The probe is rotated by 90 degrees to obtain a longitudinal plane. Identify the entrance of the IVC into the right atrium. Then the IVC diameter can be measured at one to two centimeter away from the right atrium. 

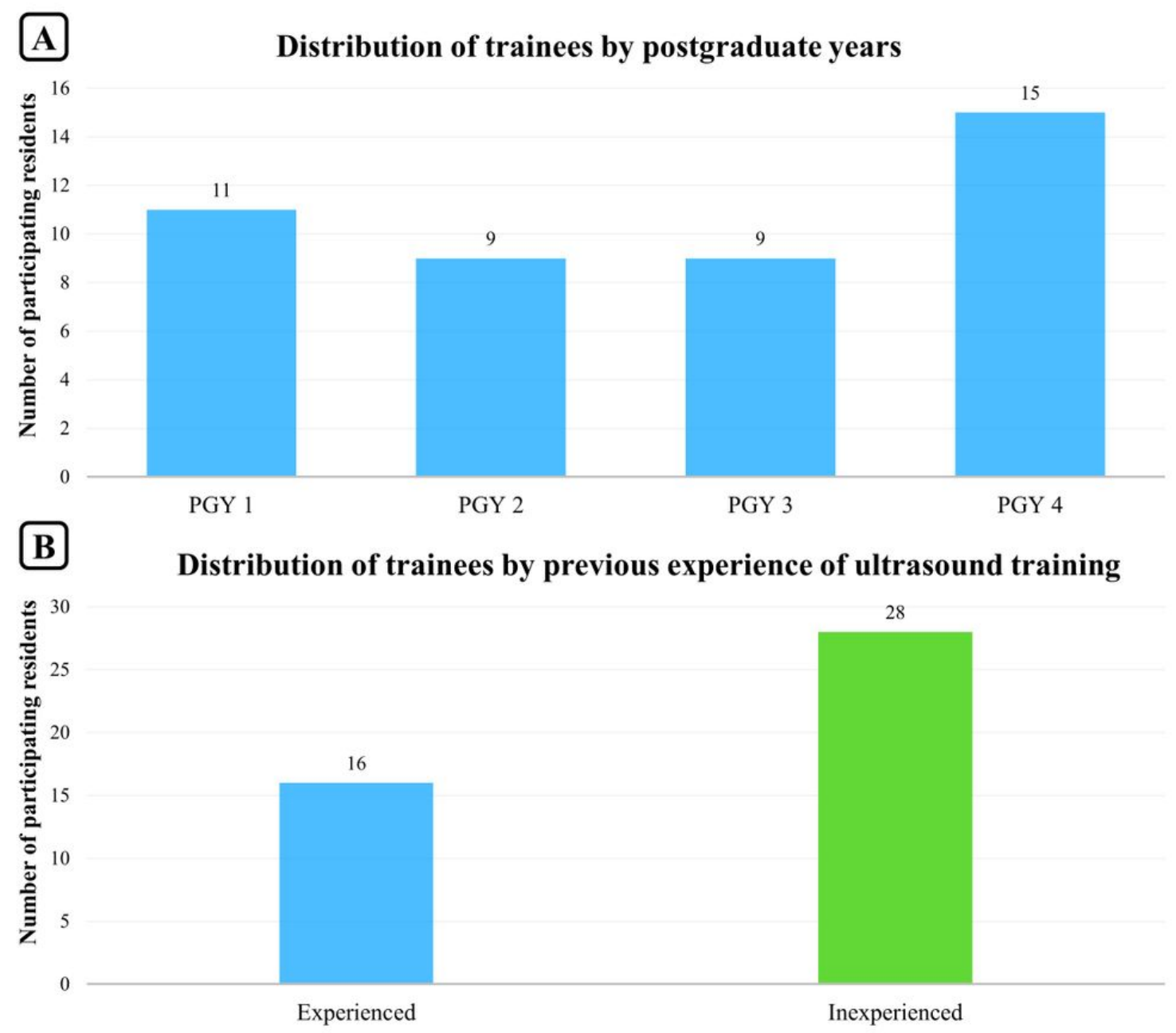

Figure 3

Demographic characteristics of enrolled trainees. A Distribution of trainees by post-graduate years. B Distribution of trainees by previous experience of ultrasound training. 


\section{Residents' self-rating score of survey question and OSAUS before and after the training program}

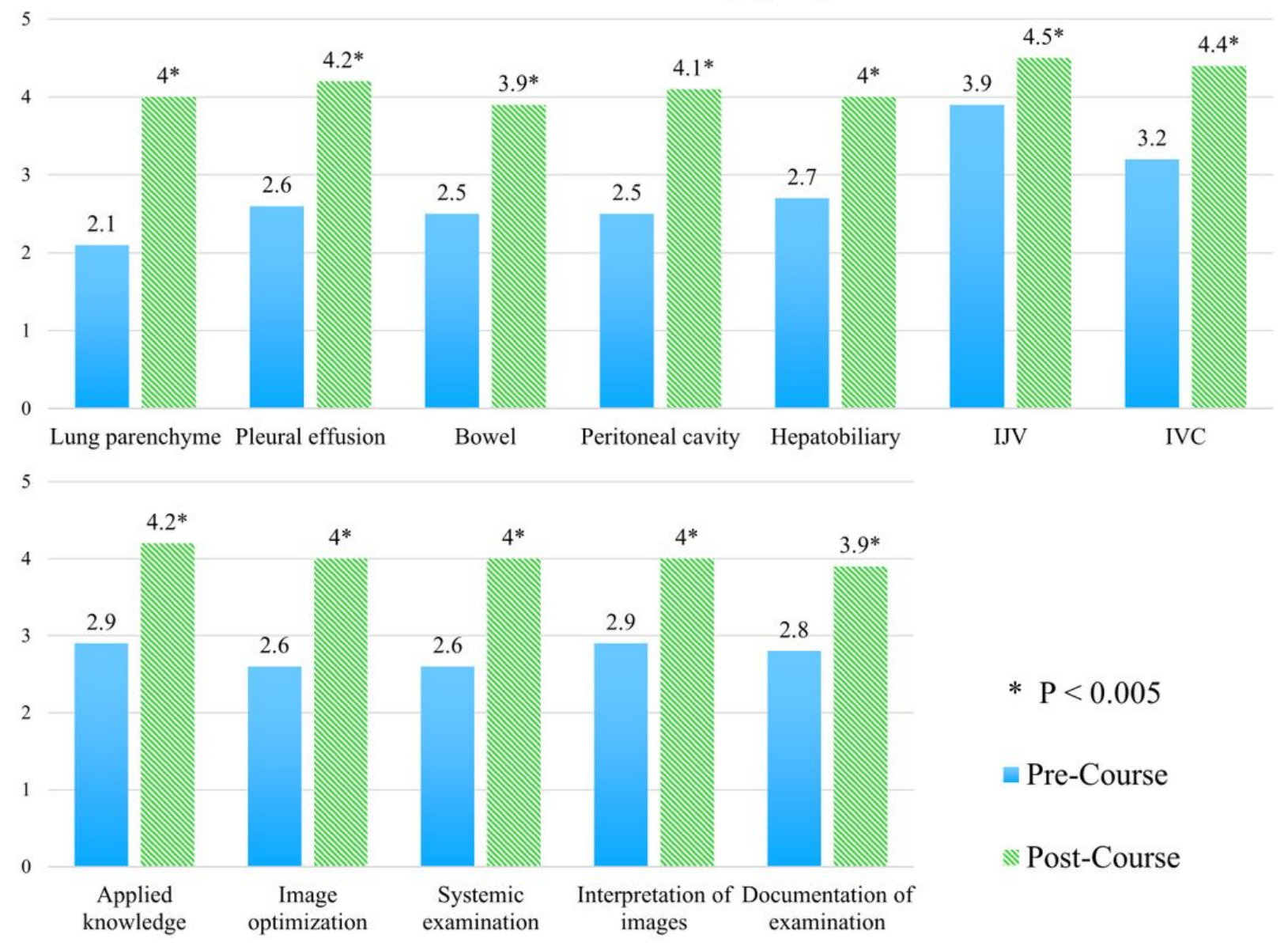

\section{Figure 4}

Residents' self-rating score of survey question and OSAUS before and after the ultrasound training program. Both scores were rated on 5-point Likert scale, where $1=1=$ not confident at all and $5=$ very confident. The blue solid bar was used for the survey question and green dashed bars were used for OSAUS.

\section{Supplementary Files}

This is a list of supplementary files associated with this preprint. Click to download.

- Additionalfile1 table1 revised0305.docx

- Additionalfile2table2revised0305.docx

- Additionalfile3table3revised0305.docx

- Additionalfile4table4revised0305.docx

- Additionalfile5Appendix1 revised0305.docx

- Additionalfile6Appendix2revised0305.docx

- Additionalfile7Appendix3revised0305.docx 